\section{ALKOHOL ALS SCHLUMMERTRUNK}

\section{Frauen sollten darauf lieber verzichten}

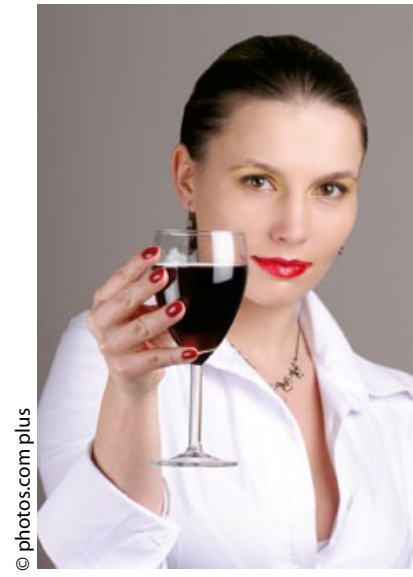

- Ein großes Glas Rotwein am Abend als Schlummertrunk - dann ist die Nachtruhe perfekt: Worauf viele Personen mit Einschlafproblemen setzen, bewirkt insbesondere bei Frauen eher das Gegenteil. Sie können zwar zunächst besser einschlafen. In der zweiten Nachhälfte wachen sie dafür häufiger auf und können länger nicht mehr einschlafen. Und am nächsten Morgen fühlen sie sich weniger ausgeschlafen als eine Vergleichsgruppe, die keinen Alkohol getrunken hat. Dies ergab eine Studie mit 93 gesunden Erwachsenen (davon 59 Frauen), die am Abend so viel Alkohol trinken sollten, bis sie in etwa 1 Promille erreicht viel für die hatten. Mit einer Polysomnografie wurde dann GesamtNachtruhe? schlafdauer, Schlafqualität, Aufwachhäufigkeiten und Dauer der nächtlichen Wachzeit erfasst. Die Wissenschaftler von der University of Michigan vermuten, dass Frauen - im Gegensatz zu Männern - den Alkohol anders verstoffwechseln und empfehlen, nicht mehr als 0,2 I Wein pro Abend zu konsumieren.

KE a

Alcohol Clin Exp Res. 2011, online first. DOI: 10.1111/j.15300277.2010.01417.x
BLUTDRUCK SENKEN, LÄNGER LEBEN

\section{Bei Frauen wird die Chance zu wenig genutzt!}

— Eine Metaanalyse liefert ein wichtiges Argument, den Blutdruck bei Frauen stärker in den Fokus zu nehmen. In der internationalen Studie war der Anteil der durch Blutdruckkontrolle vermeidbaren kardiovaskulären Ereignisse bei Frauen um 30-100\% höher als bei Männern.

Die Autoren hatten bei 9357 Männern und Frauen (mittleres Alter: 52,8 Jahre, 47\% Frauen) Blutdruckmessungen vorgenommen und sie über elf Jahre nachverfolgt. Hohe systolische Blutdruckwerte waren mit einem erheblichen Risiko für Schlaganfälle, Herzinfarkte oder die Entwicklung einer Herzinsuffizienz assoziiert. 472 Teilnehmer starben an kardiovaskulären Ursachen.

Die Frauen hatten zwar ein geringeres Ereignisrisiko als die Männer; allerdings waren bei ihnen die Blutdruckwerte mit einem steileren Anstieg der Ereignisrate verknüpft: So führte ein Anstieg von $15 \mathrm{mmHg}$ zu einer Risikoerhöhung um $56 \%$ bei Frauen, aber "nur" um 32\% bei Männern. Nach den Ergebnissen der 24-h-BlutdruckMessung wären von den kardiovaskulären Ereignissen bei den Frauen 35,9\% vermeidbar gewesen, bei den Männern 24,2\% . „Die Ergebnisse zeigen ein ungenutztes Potenzial blutdrucksenkender Maßnahmen für die kardiovaskuläre Prävention bei Frauen“, so die Autoren. EO • Hypertension online, 24. Januar 2011; DOI:10.1161/HYPERTENSIONAHA. 110.156828

\title{
HERZSTILLSTAND \\ In der Öffentlichkeit bessere Überlebenschancen?
}

— Lebensbedrohliche ventrikuläre Rhythmusstörungen, die sich „schocken“ lassen, bei denen die Patienten also von einer Defibrillation profitieren, treten signifikant öfter in der Öffentlichkeit auf als zu Hause.

Die im New England Journal of Medicine publizierte prospektive Kohortenstudie basiert auf Daten von 14059 Patienten aus dem weltweit größten Register präklinischer Herzstillstände und lebensbedrohlicher Traumata, ROCEpistry - Cardiac Arrest. Von den dokumentierten Herzstillständen, die sich an belebten Orten ereigneten, an denen Defibrillatoren angebracht waren und auch zum Einsatz kamen, waren $79 \%$ pulslose ventrikuläre Tachykardien oder Kammerflimmern. Sol- che Arrhythmien können von einem AED (Automatic external defibrillator) automatisch diagnostiziert und ggf. per Elektroschock terminiert werden. Von den häuslichen Herzstillstän-

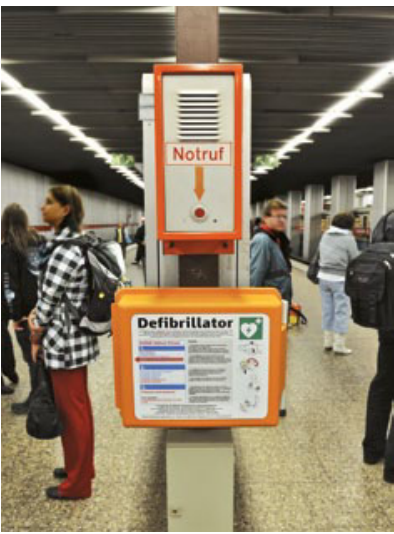

Wahrscheinlich trägt auch die größere Zahl potenzieller Nothelfer zur besseren Prognose bei.

Zeugen, die den Notruf wählen und Hilfe holen konnten.

Die höhere Anzahl von Herzstillständen, die sich "schocken“ ließen, könnte damit zusammenhängen, dass die Infarktkandidadefibrillierbaren Ereignisse 36\%. Die Chance, den Herzstillstand nach Defibrillation zu überleben, war in der Öffentlichkeit deutlich höher als zu Hause (34\% vs. 12\%). Die Forscher fanden auch, dass nur ein Drittel der Stillstände, die zu Hause eintraten, überhaupt bemerkt wurden. Dagegen gab es außerhalb der eigenen vier Wände in mehr als der Hälfte der Zwischenfälle ten, die sich in der Öffentlichkeit aufhielten, generell jünger, körperlich aktiver und gesünder waren, so die Interpretation der Studienautoren. In jedem Fall haben die Ergebnisse gezeigt, dass AEDs z.B. in Bahnhöfen, auf öffentlichen Plätzen und an anderen belebten Lokalitäten sehr wohl ihren Nutzen haben.

EO : 